\title{
Operational Procedures in the Theory of the Drug Release from Chitosan Hydrogels
}

\author{
ANDA MIHAELA CRACIUN ${ }^{1}$, GEORGIANA SERBAN², IULIA CRUMPEI ${ }^{3 *}$, MARICEL AGOP4,5, GABRIELA CIOCA ${ }^{6 *}$ \\ ${ }^{1}$ Romanian Academy of Sciences, Petru Poni Institute of Macromolecular Chemistry, Center of Advanced Research for \\ Bionanoconjugates and Biopolymers, 41A Grigore Ghica Voda Alley, 700487, Iasi, Romania \\ ${ }^{2}$ Alexandru Ioan Cuza University, Faculty of Physics, Theoretical Physics Department, 11 Carol I Blvd., 700506, Iasi, Romania \\ ${ }^{3}$ Grigore T. Popa University of Medicine and Pharmacy, Faculty of Medicine, Department of Medical Specialties (II), 16 Universitatii \\ Str. 700115, lasi, Romania \\ ${ }^{4}$ Gheorghe Asachi Technical University of lasi, Faculty of Machine Manufacturing and Industrial Management, Department of \\ Physics, 73 D. Mangeron Blvd., 700050, Iasi, Romania \\ ${ }^{5}$ Romanian Scientists Academy, 54 Splaiul Independentei Blvd., 050094, Bucharest, Romania \\ ${ }^{6}$ Lucian Blaga University of Sibiu, Faculty of Medicine, Preclinical Department, 10 Victoriei Blvd., 550024, Sibiu, Romania
}

We build a theoretical model based on a generalization of harmonic applications of Misner-type. It results a sine-Gordon type fractal differential equation whose elliptical solutions can describe, through a convenient choice of fractal dynamic constants, various modes of drug release. Thus, the entire class of empirical models (Higuchi, Korsmeyer-Peppas, Peppas-Sahlin) describing the drug release processes can be dispensed with.

Keywords: chitosan hydrogels, drug release, sine-Gordon

Local delivery of drugs is quite a new and appealing pathway for the treatment of serious diseases such as acute inflammations, tumors, osteoarthritis, periodontitis, urinary tract or ear infections [1-3]. The main advantage of the local therapy over the systemic treatment resides in the targeted release of the drug, thus limiting the side effects on normal cells [4-6]. To assure a prolonged release of the drug at the illness site, the hydrogels proved to be the most suitable materials, those based on chitosan being preferred due to their intrinsic properties such as biocompatibility and biodegradability [7].

On the other hand, despite the good properties of the chitosan which allow its safely in vivo use, the crosslinkers used for its hydrogelation proved to be fewer friendly properties, demonstrating in fact a degree of toxicity which limits their bio-applications $[8,9]$. Many attempts were pursued in order to overcome this drawback, consisting in new crosslinkers, or even in new crosslinking strategies [10-115]. An interesting approach for preparing in vivo biocompatible hydrogels was developed by hydrogelation of chitosan with citral, a natural compound extracted from lemon grass [10]. The hydrogels proved porous morphology with low diameter pores, moderate swelling in physiological $\mathrm{pH}$ while their shape was well preserved, these being important features for local therapies. To this respect, the hydrogels were used as matrix for the encapsulation of an antitumor model drug, 5-Fluorouracil (5FU), and indicated an obvious improvement of the release in terms of increasing the drug efficiency by its prolonged delivery, minimizing the side effects [16].

These positive results encouraged us to go further in the understanding of the release process, by building a theoretical model based on a generalization of harmonic applications of Misner-type, capable of providing new insights of the drug release theoretical modeling.

\section{Experimental part \\ Materials}

Chitosan of low molecular weight, citral of $95 \%$ purity, 5-Fluorouracil of purity higher of $99 \%$, and phosphate buffer solution were purchased from Aldrich and used as received.

\section{Drug delivery systems preparation}

The drug delivery systems investigated in this paper were prepared by in situ hydrogelation of the chitosan with citral, in the presence of 5-Fluorouracil. The synthetic procedure can be resumed as follows. Chitosan was dissolved in a $0.7 \%$ acetic acid to form a $3 \%$ solution. The solution was warmed up to $55^{\circ} \mathrm{C}$ and kept at this temperature under vigorous stirring. Targeting the simultaneous hydrogelation and drug encapsulation, a solution of citral and 5fluorouracil dissolved together in a water/ethanol mixture $(1 / 1, v / v)$ was slowly dropped upon the chitosan solution. The amount of drug has been kept constant, while the ratio of the glucosamine units of chitosan and citral was varied in order to reach hydrogels with different crosslinking densities. The amounts of the reagents and the codes of the resulted hydrogels are given in figure 1.

\section{The in vitro drug release protocol}

The in vitro drug release has been monitored in vitro, simulating in vivo physiological conditions, consisting mainly in the use of phosphate buffer solution of $\mathrm{pH}=7.4$ (PBS) as release medium and keeping a constant temperature of $37^{\circ} \mathrm{C}$ on the entire period of investigation. The CF1-CF4 samples of similar weight were immersed into vials containing $10 \mathrm{~mL}$ of PBS. From time to time, 2 $\mathrm{mL}$ of supernatant was extracted and replaced with pure PBS. All the supernatant samples were collected and further analysed by UV-Vis spectroscopy, by measuring the 5FU 


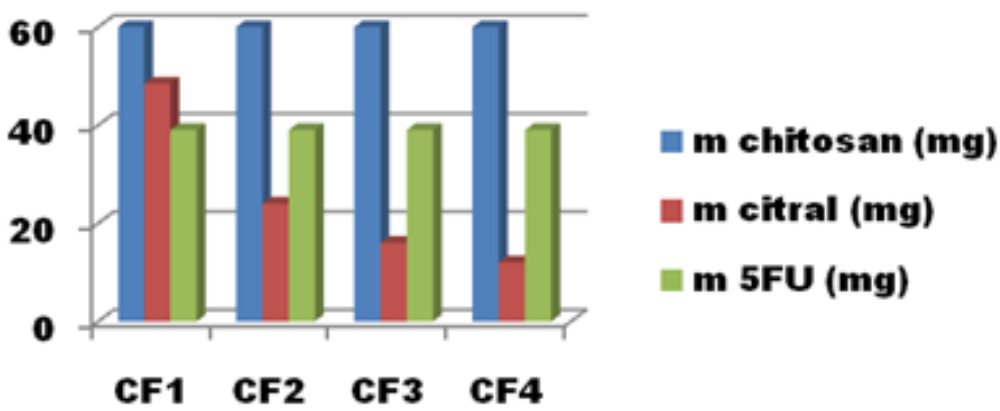

Fig. 1. Graphical representation of the amounts of the hydrogel components

absorbance. The resulted data were fitted on the calibration curve of the 5FU drug, which was traced before. It should be mentioned that the calibration curve of 5FU was obtained by graphic representation of the absorbance against known concentrations, using the absorption maximum from its spectrum, at $265 \mathrm{~nm}$ [16-18]. The cumulative release of the 5FU was calculated using Lambert-Beer law. The experiments were performed in triplicate. Absorbance of the released drug from collected samples was measured using a Horiba Spectrophotometer.

The release kinetics of the systems was studied by fitting the obtained release data on the following models [19,20]:

-Zero order model: $\mathrm{Q}_{t}=\mathrm{K}$. $\mathrm{t}$ where $\mathrm{Q}_{t}$ is the amount of drug dissolved in the time tand $K_{0}$ is the zero order release constant.

-Higuchi model:: $\mathrm{Q}_{t}=\mathrm{K}_{H}, \mathrm{t}^{1 / 2}$, where $\mathrm{Q}_{\mathrm{t}}$ is the amount of drug released in the time tand $K_{H}$ is the Higuchi dissolution constant.

-Korsmeyer-Peppas model: $\frac{M_{t}}{M_{\infty}}=K \cdot t^{n}$, where $\frac{M_{t}}{M_{\infty}}=K \cdot t^{n}$ is the fraction of drug released at the time $t$, $K$ is the release rate constant and $n$ is the release exponent.

\section{Results and discussions}

The drug delivery systems prepared for this study proved a porous morphology with evident drug crystals in the case of the samples with high crosslinking density (CF1, CF2), fact attributed to the high viscosity of the systems which hampered the drug dispersion and thus favored its crystallization (fig. 2). On the other hand, the CF3 and CF4 samples with lower crosslinking density showed no obvious segregation of the drug, indicating its fine dispersion into the hydrogel matrix.

The monitoring of the 5FU release from the under study systems was represented in figure 3 . As it can be seen, the release was significantly affected by the encapsulation pathway. From CF1 and CF2 systems, in which the 5FU drug was confined as micrometric crystals, the release occurred faster, while from CF3 and CF4 systems, in which the drug was fine dispersed, the release was slower. The encapsulation pathway played a key role in the total amount of drug released from the matrix, too. As it can be seen, after 32 hours, the CF1 released almost the entire amount of drug, while the CF 4 only $75 \%$. This can be explained by stronger intermolecular forces developed in the case of the fine dispersion of the drug into the hydrogel matrix, forces which surpassed the interfacial ones developed in the case of dispersion of the drug as microcrystals [2126].

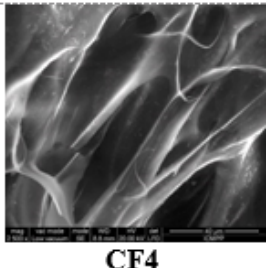

Fig. 2. Representative SEM images of the under study drug delivery systems
Fig.3. Graphical representation of the 5FU release from CF1-CF4 systems 


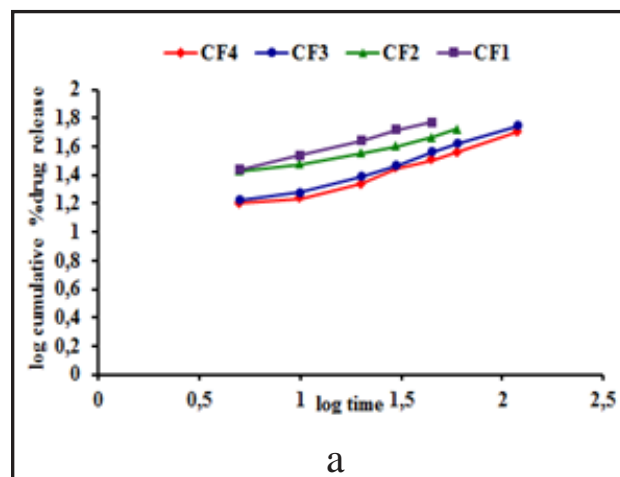

To further understand the kinetics release of the $\mathbf{5 F U}$ drug from the CF1-CF4 systems, the release data were fitted on the mathematical equations of the KorsmeyerPeppas, Higuchi and Zero Order models (fig. 4).

As it can be seen in Figure 4, the obtained release data proved a good fitting on the Korsmeyer-Peppas and Higuchi models, indicating that the release mechanism is controlled by the diffusion of the 5FU, first from the superficial layers of the hydrogel, follow ed by its diffusion form bulk to outside [19, 27].

\section{Theoretical considerations}

As spinons are considered fundamental for the spacetime structure [22], so we can admit that $2 \times 2$ real matrices

$$
\hat{\boldsymbol{\alpha}}=\left(\begin{array}{ll}
\alpha & \beta \\
\gamma & \delta
\end{array}\right)
$$

become relevant to certain types of dynamics in the polymeric matter. The elements of this matrix contain, yet in an unspecified form, both the physical parameters of the polymeric matter implied in an evolution and the possible initial conditions of this evolution.

Any matrix $2 \times 2$ given by (1) can be written as a linear combination with real coefficients involving two special matrices, namely unit matrix 1 and a matrix of null trace $\mathbf{i}$ (an involution) of the form:

$$
\hat{\boldsymbol{\alpha}}=\lambda \hat{\mathbf{l}}+\mu \hat{\mathbf{i}}
$$

Involution $\mathbf{i}$ has the properties that: (a) its square is multiple of 1 and $(b)$ the fixed points of its homographic action are those of the matrix $\boldsymbol{\alpha}$. In equation (2) we have the freedom to choose a parameterization in which $\mathrm{i}$ square's to be right the unitmatrix up to a sign. In this case, we can express i elements only by two parameters representing the asymptotic directions of the matrix $\alpha$. If these parameters are complex, then the anharmonic curves generated by $\alpha$ according to prescription $[29,30]$

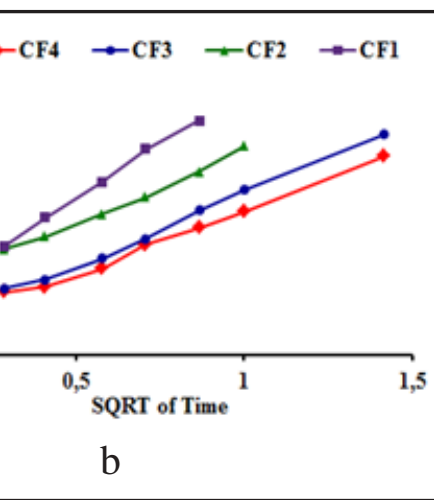

Fig 4. Linear forms of KorsmeyerPeppas (a) and Higuchi (b) models applied for the release of $5 \mathrm{FU}$ from the CFU hydrogels are logarithmic spirals, while those generated by involution $i$ are ellipse. In this case, we are entitled to discuss the interactions in connection with the forms of polymeric matter, geometrically describe. The procedure is the following.

In the case of complex asymptotic directions, the representation of the matrix $\mathbf{i}$ through the asymptotic directions is a spherical one. It is completely analogous to the spherical representation of vectors in the Euclidian case. The difference lies only in the fact that the role played by the vector magnitude is now taken over by the matrix determinant. If the asymptotic directions are, let's say, $(u+i v)$, then the spherical representation satisfying the above conditions (a) and (b) is:

$$
\mathbf{i}=\frac{1}{\mathrm{v}}\left(\begin{array}{cc}
-u & -u^{2}-v^{2} \\
1 & u
\end{array}\right) ; \quad \mathbf{i}^{2}=-\mathbf{l}
$$

In the usual case of the self-structuring of any polymeric matter, $u$ and $v$ can be expressed in relation to the initial conditions of the evolution [31]. The evolutions, of which these initial conditions are chosen, must be, accordingly, of the same nature as the current evolution which it characterizes by the dynamic equations.

Therefore, the essential idea is that the elements of real matrices $2 \times 2$ represent, through the associated ansatz [31], polymeric matters in evolution. Such a representation of polymeric matter has the advantage of allowing an explicit differential description of polymeric matter itself, through metric geometry, just like the metric description of space.

Indeed, the representation of polymeric matter through $2 \times 2$ matrices leads to a natural metric of the matrices space, for example the Killing-Cartan metric of the SL(2R) algebra of these matrices [31,32]. The base co-vectors of such a geometry are given, in the general case of a matrix (1), by differential forms.

These differential forms in the parameterization given in equations (2) and (3) are:

$$
\begin{aligned}
& \omega^{1}=\frac{1}{v} d \phi+\sin ^{2} \phi \frac{d u}{v^{2}}-\sin \phi \cos \phi \frac{d v}{v^{2}} ; \\
& \omega^{2}=2 \frac{u}{v} d \phi+2 \sin ^{2} \phi \frac{u d u+v d v}{v^{2}}+2 \sin \phi \cos \phi \frac{v d u-u d v}{v^{2}} ; \\
& \omega^{3}=\frac{u^{2}+v^{2}}{v} d \phi+\sin ^{2} \phi \frac{\left(u^{2}-v^{2}\right) d u+2 u v d v}{v^{2}}+\sin \phi \cos \phi \frac{2 u v d u-\left(u^{2}-v^{2}\right) d v}{v^{2}}
\end{aligned}
$$

where $\tan \phi=\phi \mu / \lambda$. In relation to these co-vectors, the metric is given by the square form:

$$
d s^{2}=\omega^{1} \omega^{3}-\left(\omega^{2} / 2\right)^{2}=d \phi^{2}-\sin ^{2} \phi \frac{d u^{2}+d v^{2}}{v^{2}}
$$

One can conclude that, at least as long as the polymeric matter is represented by the property that physics admits as essential, namely the evolution, its description mode is a metric geometry. In this case, the metric is given in equation (5), where $\phi$ is an arbitrary phase angle, and $u$ and $v$ are coordinates obtained from the (local) dynamics of the polymeric matteras described above. Such a connection can definitely be delegated to a generalization of harmonic applications of 
Misner type [33]. As soon as we have solved in this manner the application way of the polymeric matter on the space that it has, the quantities $\phi, u, v$ (and thus the elements of the matrix family representing the polymeric matter) are obtained, in principle, as functions of the fractal coordinates which makes it possible to accurately define a density of polymeric matter in space (without appealing to the usual uncontrollable assumptions about the expansion of polymeric matter, the space expansion or the existence of space-time, etc.)

We are now forced to establish the connection polymeric matter - space and backward. To illustrate the procedure, let us take the case of metric (5) for the polymeric matter, admitting, in a cartanian sense, a space metric in the fractal coordinates $x^{\prime}(t, d t)$, with ttime and $d t$ the scale resolution [33-35]. The principle of harmonic application allows us to always find the material coordinates $(\phi, u, v)$ as functions of fractal coordinates, showing how the structured polymeric matter is distributed in space. The volume element of the metric (5) is the 3-differential form:

$$
d M \equiv \frac{\sin ^{2} \phi}{v^{2}} d \phi \wedge d u \wedge d v
$$

while the volume element of space in a fractal mentality has the form $d V \equiv d x^{1} \wedge d x^{2} \wedge d x^{3} \wedge$ with the exterior product. The notation from equation (6) suggests that we have here a mass, because the material coordinates reflect a dynamic problem. Once we have these coordinates, it is easy to calculate a density, as a ratio between the volume element of the polymeric matter, whether structured or not, and the volume element of the space. Formally, we have for the density of polymeric matter in space, the relation:

$$
\rho \equiv \frac{d M}{d V}=\frac{\sin ^{2} \phi}{v^{2}} J(\phi, u, v ; \mathbf{x})
$$

where $J(\phi, u, n ; x)$ represents the Jacobian of transformation $\left(x^{1}, x^{2}, x^{3}\right) \stackrel{\rightarrow}{\rightarrow}(\phi u, v)$ thatwe eventually discovered through the principle of harmonic application.

The problem of representation of polymeric matter in this scheme is now solved by the harmonic application from space to polymeric matter $x \rightarrow \xi$ that gives us the density from equation (7) as function of coordinates. Thus, we consider the fractal functional of the principle of harmonic application:

$$
\mathbf{I} \equiv \frac{1}{2} \iiint\left(d^{3} \mathbf{x}\right) \sqrt{|h|} h^{i j}(\mathbf{x}) \frac{\partial \xi^{\mu}}{\partial x^{i}} \frac{\partial \xi^{v}}{\partial x^{j}} g_{\mu \nu}(\xi) \text { (8) }
$$

where $\mathbf{h}$ is the metric of space, and $\mathbf{g}$ is the metric of polymeric matter. The cancellation of the first order variation of this functional, relative to the fractal coordinates, gives us the harmonic application we are looking for. Given that the space is fractal and using the equation (5) for the metric tensor in polymeric matter, we must have for the integrant of equation (8) the expression:

$$
\sqrt{|h|} h^{i j}(\mathbf{x}) \frac{\partial \xi^{\mu}}{\partial x^{i}} \frac{\partial \xi^{v}}{\partial x^{j}} g_{\mu v}(\xi) \equiv(\nabla \phi)^{2}-\left(\frac{\sin \phi}{v}\right)^{2}\left[(\nabla u)^{2}+(\nabla v)^{2}\right] \text { (9) }
$$

where we use the notation $\nabla$ for the gradient in fractal coordinates. The Euler equations corresponding to the functional (8) are:

$$
\begin{aligned}
& \nabla^{2} \phi+\sin \phi \cos \phi \frac{(\nabla u)^{2}+(\nabla v)^{2}}{v^{2}}=0 \\
& \nabla\left(\frac{\nabla u}{v^{2}}\right)=0 ; \nabla\left[\left(\frac{\nabla u}{v^{2}}\right)+\frac{(\nabla u)^{2}+(\nabla v)^{2}}{v^{2}}\right]=0
\end{aligned}
$$

The last two equations represent a harmonic application from the fractal space to the fractal hyperbolic plane. As a consequence of those equations we have, as one can easily verify, the equation:

$$
\nabla \frac{(\nabla u)^{2}+(\nabla v)^{2}}{v^{2}}=0
$$

This means that the scalar quantity under the differential gradient operator is constant, real and always positive, being a sum of two squares of some real quantities. Let us note that this quantity with $\mathrm{m}^{2}$, where $\mathrm{m}$ is real. The first equality from (10) becomes the sine-Gordon equation of the fractal type:

$$
\nabla^{2} \phi+m^{2} \sin \phi \cos \phi=0
$$

A solution of this equation can be simply given if we assume that the quantities depend on $x^{i}$ through the linear form:

$$
\xi=a_{1} x^{1}+a_{2} x^{2}+a_{3} x^{3}
$$

In this case, the equation (12) becomes:

$$
\phi^{\prime}+\frac{m^{2}}{a^{2}} \sin \phi \cos \phi=0
$$

where the accent notes the derivative after $\xi$ and $a^{2}$ is the square of the vector a. Further, the equation (14) is multiplied with $\phi^{\prime}$, after which it can be integrated and leads to:

$$
\phi^{\prime 2}+\frac{m^{2}}{a^{2}} \sin ^{2} \phi=c^{2}
$$

where $c$ is an integration constant that we assume real. Therefore, $\xi$ is the elliptic integral of first order [35]:

$$
c\left(\xi-\xi_{0}\right)= \pm \int_{0}^{\phi} \frac{d \phi}{\sqrt{1-k^{2} \sin ^{2} \phi}} ; k^{2} \equiv \frac{m^{2}}{c^{2} a^{2}}
$$

So, for $z=\sin \phi$ the inverse of the integral (16) written in the form:

$$
c\left(\xi-\xi_{0}\right)= \pm \int_{0}^{\operatorname{arcsinz}} \frac{d z}{\sqrt{\left(1-z^{2}\right)\left(1-k^{2} z^{2}\right)}}
$$

involves sn Jacobi's elliptic function of module $k$,

$$
z=\operatorname{sn}\left[c\left(\xi-\xi_{0}\right) ; k\right]
$$

In the previous relations $c, \xi$, and k are fractal constants (dependent on scale resolution). As by the connection between polymeric matter -space and backwards, the metric element (5) is preserved between the two varieties, the one associated to the polymeric matter through the coordinates $(u, v, \phi)$ and the one associated to space through the fractal coordinates $\left(x^{1}, x^{2}, x^{3}\right)$, isometry (immersions, compacti-fication, etc.) can be established. In such a context, $z$ can be matched with an adimensional variable linked to the cumulative drug release in the form $z=m(t) / m_{\alpha}$, while $c\left(\xi_{-} \xi_{0}\right)$ with the temporal adimensional coordinate in the form $c\left(\xi-\xi_{0}\right)=\Omega\left(t-t_{0}\right)$.

Then, by a proper normalization, equation (18) can be written in the form:

$$
m(t)=m_{\mathrm{w} s} n\left\lfloor\Omega\left(t \quad t_{0}\right) ; k\right]
$$

the various scale resolutions being imposed by the module of sn function (fig. 5). The curves of figure 2 can be obtained as degenerations of the sn elliptic function for $k$ values close to 1 , as can be seen in figure 6 . 


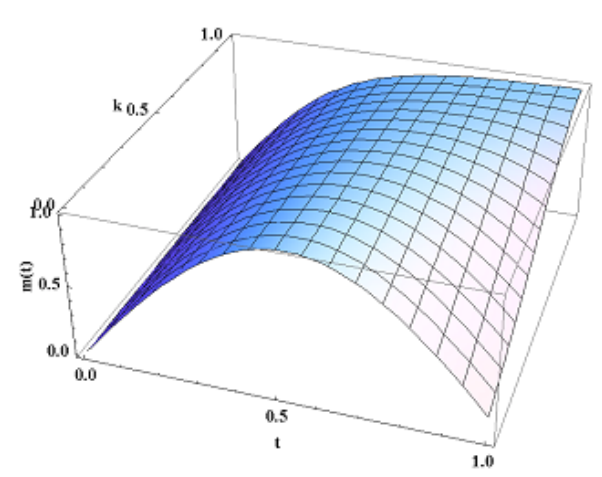

Fig. 5. Three dimensional dependence of the drug release amount on time $t$ and fractal constant $k$
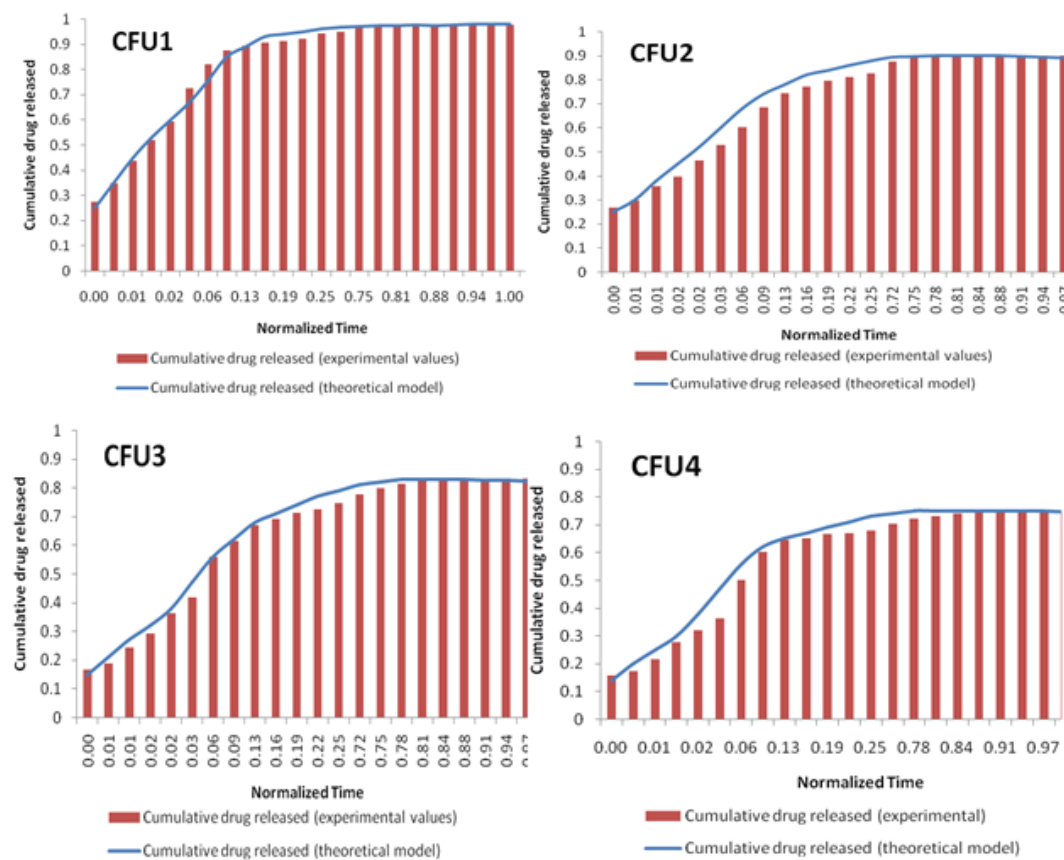

Fig. 6. Comparative plots (experimental vs. theoretical) for cumulative drug release

\section{Conclusions}

We build a theoretical model based on a generalization of harmonic applications of Misner-type. It results a sineGordon-type fractal differential equation whose elliptical solutions can describe, through a convenient choice of fractal dynamic constants, various modes of drug release. Thus, the entire class of empirical models (Higuchi, Korsmeyer-Peppas, Peppas-Sahlin) describing the drug release processes can be dispensed with.

Acknowledgements: This work was supported by European Union's Horizon 2020 research and innovation programme (grant agreement 667387) and Romanian National Authority for Scientific Research MEN - UEFISCDI (grant number PN-III-P4-ID-PCCF-2016-0050).

\section{References}

1.PEREIRA, G. G., LAWSON, A. J., BUTTINI, F., SONVICO, F., Drug Deliv., 23, 2016, p. 2881

2.OLARU, A. M., MARIN, L., MORARIU, S., PRICOPE, G., PINTEALA, M., TARTAU-MITITELU, L., Carbohydr. Polym., 179, 2018, p. 59

3.SEDGHI, R., SHAABANI, A., MOHAMMADI, Z., SAMADI, F. Y., ISAEI, E., Carbohydr. Polym., 159, 2017, p. 1

4.WOLINSKY, J. B., COLSON, Y. L., GRINSTAFF M.W., J. Control. Release, 159, 2012, p. 14

5.FODOR, K., TIT, D. M., PASCA, B., BUSTEA, C., UIVAROSAN, D., ENDRES, L., IOVAN, C., ABDEL-DAIM, M., BUNGAU, S., Oxid. Med. Cell. Longev., 2018, 2018, ID 4147320, https://doi.org/10.1155/2018/ 4147320

6.ABDEL-DAIM, M. M., ZAKHARY, N. I., ALEYA, L., BUNGAU, S. G., BOHARA, R. A., SIDDIQI, N. J., Oxid. Med. Cell. Longev., 2018, 2018, ID 2098123, 2018, https://doi:10.1155/2018/2098123

7.MARIN, L., AILINCAI, D., MARES, M., PASLARU, E., CRISTEA, M., NICA, V., SIMIONESCU, B. C., Carbohydr. Polym., 117, 2015, p. 762 8.BEAUCHAMP, R.O., ST. CLAIR. M. B., FENNELL, T. R., CLARKE, D. O., MORGAN, K. T., Crit. Rev. Toxicol., 22, 1992, p. 143

9.CARAC, A., BOSCENCU, R., DEDIU, A. V., BUNGAU S. G., DINICA R. M., Rev. Chim.(Bucharest), 68, no. 7, 2017, p. 1423.

10.MARIN, L., AILINCAI, D., MORARIU, S., TARTAU-MITITELU, L., Carbohydr. Polym., 170, 2017, p. 60

11.IFTIME, M., MORARIU, S., MARIN, L., Carbohydr. Polym. 165, 2017, p. 39

12.ROTARU, A., COJOCARU, C., CRETESCU, I., PINTEALA, M., TIMPU, D., SACARESCU, L., HARABAGIU, V., Sep. Purif. Technol., 133, 2014, p. 260

13.AILINCAI, D., MARIN, L., MORARIU, S., MARES, M., BOSTANARU, A.C., PINTEALA, M., SIMIONESCU, B.C., BARBOIU, M., Carbohydr. Polym. 152, 2016, p. 306

594
14.BEJ AN, A., AILINCAI, D., SIMIONESCU, B. C., MARIN, L., Polym. Chem. 9, 2018, p. 2359

15.IFTIME, M. M., MARIN, L., Ultrason. Sonochem., 45, 2018, p. 238 16.AILINCAI, D., MITITELU, TARTAU, L., MARIN, L., Drug Deliv., 25, 2018, p. 1080

17.FIFERE, A., MARANGOCI, N., MAIER, S., COROABA, A., MAFTEI, D., PINTEALA, M., Beilstein J. Org. Chem., 8, 2012, p. 2191

18.SZAJ DZINSKA-PIETEK, E., PINTEALA, M., SCHLICK, S., Polymer, 45, 2004, p. 4113

19.BACAITA, E. S., CIOBANU, B. C., POPA, M., AGOP, M., DESBRIERES, J., Phys. Chem. Chem. Phys., 16, 2014, p. 25896

20.BACAITA, E. S., AGOP, M., Phys. Chem. Chem. Phys., 18, 2016, p. 21809

21.MARIN, L., POPESCU, M.C., ZABULICA, A., UJI, H., FRON, E., Carbohydr. Polym. 95, 2013, p. 16

22.PAUN, V. A., POPA, M., DESBRIERES, J., PEPU, C. A., DRAGAN, S. V., ZEGAN, G., CIOCA, G., Mat. Plast., 53, no. 4, 2016, p. 590.

23.PAUN, V. A., OCHIUZ, L., HORTOLOMEI, M., CRETEANU, A., STOIERIU, I., GHICIUC, C. M., SERBAN, G. T., ZEGAN, G., CIOCA, G., Mat. Plast., 53, no. 4, 2016, p. 699.

24.MOROSINI, C., MARSONI, M., TORRETTA, V., CONTI, F., RAGAZZI M., RADA, E., CIOCA, G., Sustainability, 9, no. 8, 2017, doi: 10.3390/ su9081466

25.CIOCA, G. BACAITA, E.S., AGOP, M., URSULESCU LUPASCU, C., Comput. Math. Meth. Med., 2017, 2017, doi: 10.1155/2017/5748273

26.CSOKMAI, L. S., TARCA, R.C., BUNGAU, C., HUSI, G., Int. J . Comput. Commun. Control, 10, no. 1, 2015, p. 30.

27.CIOCA, G., AGOP, M., POPA, M., BUNGAU, S., BUTUC, I., Rev. Chim.(Bucharest), 68, no. 12, 2017, p. 2925.

28.BACAITA, E. S., RADU, V., ULINIUC, A., POPA, M., SUSANU, S., Mat. Plast., 50, 2013, p. 18

29.PENROSE, R., Ann. Phys., 10, 1960, p. 171

30.MIHAILEANU, N., Complements of Geometry: Analytical, Projective and Differential, Didactic and Pedagogic Publishing House, Bucharest, 1972

31.MAZILU, N., AGOP, M., Skyrmions. A great Finishing Touch to Classical Newtonian Philosophy, Nova Publishers, New York, 2012 32.MISNER, C. W, Phys. Rev. D, 18, 1978, p. 4510

33.NOTTALE, L., Scale Relativity and Fractal Space-Time. A new approach to unifying Relativity and Quantum mechanics, Imperial College Press, London, 2011

34.COSTAN, A., DIMA, A., IONITA, I., FORNA, N., PERJU, M. C., AGOP, M., Optoelectron. Adv. Mater. - Rapid Commun., 5, 1-2, 2011, p. 92

35.URSU, C., POMPILIAN, O., GURLUI, S., NICA, P., AGOP, M., DUDECK, M., FOCSA, C., Appl. Phys. A -Mater. Sci. Process., 101, 1, 2010, p. 153 Manuscript received: 18.10 .2018 